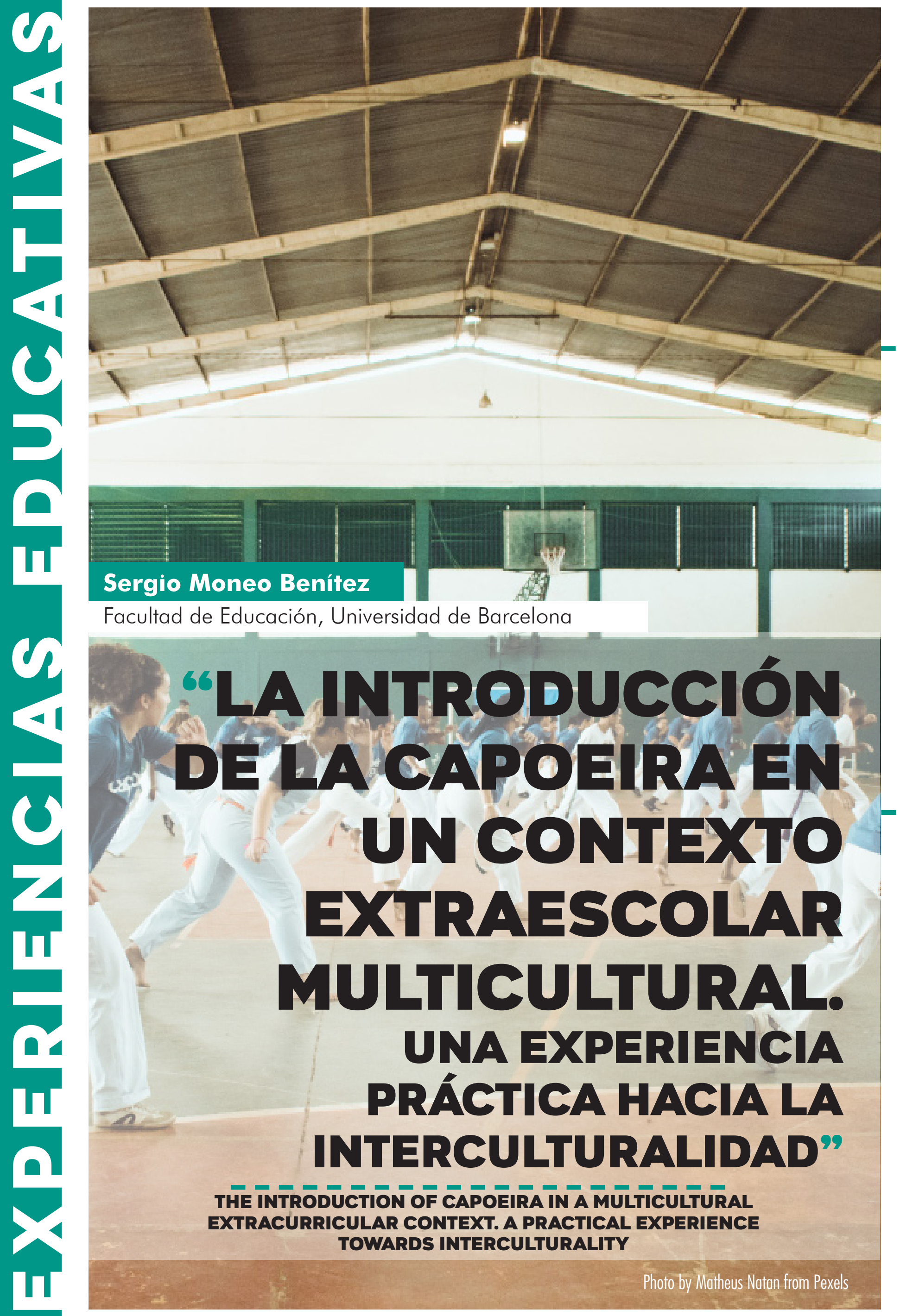




\section{RES UMEN}

Mediante este artículo se pretende mostrar cómo es posible fomentar la interculturalidad en un contexto de educación no formal, o extraescolar, introduciendo la actividad física de la Capoeira. A tal efecto, se presenta parte de un estudio que aborda la problemática social en la que se encuentra un centro escolar debido, en esencia, a la segregación que existe entre sus alumnos y alumnas ', la cual está causada por sus diferencias culturales, lingüísticas, nativas, etc. Éste es un estudio de casos en el que el diseño y la aplicación de una propuesta didáctica, vinculada con la Capoeira, permite potenciar los vínculos personales de 27 participantes (comprendidos en una edad de entre 10 y 12 años) que se encuentran situados en la problemática social mencionada, así como un comportamiento apropiado en sus prácticas grupales. La información del proceso es obtenida a partir de la observación participante y de los registros en vídeo de las 39 sesiones extraescolares llevadas a cabo, la cual se recoge en un diario de campo, y de las entrevistas realizadas a distintos informantes: los 2 monitores que dirigieron el proceso empírico, las tutoras del alumnado perteneciente a los grupos A y B del centro escolar y el maestro de Educación Física de la escuela.

\section{PALABRAS CLAVE}

\section{Multiculturalidad, Interculturalidad, Educación no formal, Propuesta dídáctica, Capoeira}

\section{A B STRACT}

This article aims to show how it is possible to promote interculturality in a context of non-formal or extra-curricular education, introducing physical activity in Capoeira. To this end, part of a study that addresses the social problem in which a school is found is presented, due, in essence, to the segregation that exists between its students, which is caused by their cultural, linguistic differences, native, etc. This is a case study in which the design and application of a didactic proposal, linked to Capoeira, enables the personal ties of 27 participants (aged between 10 and 12 years) who are located in the social problems mentioned, as well as appropriate behavior in their group practices. The information of the process is obtained from the participant observation and from the video records of the 39 extracurricular sessions carried out, which is collected in a field newspaper, and from the interviews carried out with different informants: the 2 monitors who They directed the empirical process, the tutors of the students belonging to groups $A$ and $B$ of the school and the Physical Education teacher of the school.

\section{KEYWORDS}

Multiculturalism, Interculturality, Non-formal education, Didactic proposal, Capoeira

\footnotetext{
1 Para facilidad del lector, en adelante se usará el témino masculino en referencia a ambos géneros.
} 


\title{
LA INTRODUCCIÓN DE LA CAPOEIRA EN UN CONTEXTO EXTRAESCOLAR MULTICULTURAL. UNA EXPERIENCIA PRÁCTICA HACIA LA INTERCULTURALIDAD
}

- - - - - - - - - - - - - - - - - - - - - -

\author{
Sergio Moneo Benítez
}

Facultad de Educación, Universidad de Barcelona

\section{INTRODUCCIÓN}

La interculturalidad surge en aquellos entornos en los que cohabitan personas de rasgos culturales distintos. Por este motivo, su desarrollo solo es posible en un contexto multicultural (Barabas, 2014). La convivencia en la pluralidad le ofrece al ciudadano la posibilidad de conocer diversos modos de vivir en sociedades con diversidad. Berttollini y Langón (2009, p.41) determinan que esta diversidad se encuentra en acciones, a priori poco trascendentes (formas de saludar o de vestir), así como en aspectos potencialmente relevantes (opciones sobre un criterio moral). Para comprender modos de vida distintos, son necesarias la interacción y la comunicación entre sujetos de características diversas; procesos que surgen al reconocer y comprender las culturas que existen (Bernabé, 2012) con respeto, igualdad y tolerancia.

Subyace aquí el pluralismo cultural el cual, para Giménez (2003), se basa en los principios de igualdad (o no discriminación) y de diferencia (o respeto y aceptación del Otro). Desde la pedagogía, la educación intercultural amplia la formación del alumnado en este plano y promueve la creación de vínculos sociales sin atender a diferencias entre ciudadanos. Aplicar estos principios en cualquier realidad multicultural, ayudaría a que todo habitante tuviera mismos derechos, responsabilidades e igualdad de oportunidades.

De esta forma, pluralismo cultural y educación intercultural equiparan posturas en cuanto a unión ciudadana y eliminación de factores excluyentes como origen, idioma o ideología. Así, cuando nativos e inmigrantes adquieren el compromiso de convivir en una misma sociedad global, es viable la diversidad en la pluralidad. En la actualidad, sin embargo, es utópico para la mayoría de sociedades y los comentarios (reiterados) entre habitantes se deben, como indica Bernabé (2016, p.330):

a que todavía no se ha conseguido la situación intercultural que la legislación educativa defiende; y que por ello es necesario seguir insistiendo en este tipo de temática, para mover a la reflexión (y a la acción) en los correspondientes ámbitos educativos.

Gran parte de la población asume, de modo natural e instintivo, que la cultura primaria de una sociedad pluricultural es la que prevalece sobre las foráneas y, más aún, ante aquéllas de recién llegada. El ciudadano nativo del propio lugar adquiere un estatus superior que, en algún momento durante su encuentro, manifiesta ante el extranjero; una situación frecuente en colectivos que cohabitan en un mismo territorio y que representan diversidad. Berttollini y Langón (2009, p.42), aluden a este hecho afirmando que las diferencias no pasan desapercibidas:

El contacto intercultural, la revelación del "otro" como distinto, no es nunca un acontecimiento irrelevante para la conciencia individual o colectiva. Siempre conmociona profundamente y tiene consecuencias revulsivas en cada una de las áreas culturales que se confrontan. 
En este sentido, los principios de igualdad y de diferencia son un compromiso de todos. En el caso del inmigrante, asume su responsabilidad para adaptarse a la sociedad; hecho que a veces no sucede e, incluso, propicia que el ciudadano residente se vaya. En conjunto, se vislumbra la necesidad de fomentar una convivencia sana en una sociedad diversa. Porras (2010, p. 14) indica al respecto:

La educación multicultural se presenta en la actualidad como una problemática apremiante, tanto en lo que respecta a la inserción de las minorías étnicas como de los inmigrantes en una comunidad cualquiera.

En definitiva, comprender y compartir la cultura se fundamenta en el respeto y la tolerancia hacia todo ciudadano, sin atender a diferencias y con mutuo compromiso; de esta manera progresará. Toda sociedad será intercultural cuando exista una interacción y comunicación entre sus habitantes (Bernabé, 2012).

\section{MARCO DE REFERENCIA}

Pronto se cumplirán dos décadas desde que los ciudadanos nos adentramos en el siglo XXI, un tiempo globalizado en el que la sociedad está formada por ciudadanos con origen distinto. El inicio de este fenómeno se halla en la corriente migratoria producida en los últimos años del siglo XX y que, de forma natural, ha transformado pensamiento y convivencia (García, Escarbajal Frutos y Escarbajal De Haro, 2007). Esta coexistencia entre residentes y forasteros generó el uso de términos referentes a diferentes ideologías, corrientes y/o comportamientos que se encontraban en una misma zona. Con el tiempo, el aumento de la población inmigrante ha sido notorio (Vecina, 2013), lo cual ha hecho que los conceptos "multicultural" e "interculturalidad" experimenten un nuevo auge en la ciudadanía y actualmente posean un gran protagonismo (Barabas, 2014). No obstante, a menudo se confunden y se utilizan de modo erróneo, o impreciso, debido a sus rasgos comunes (García, 2005). Por su relevancia en este estudio, es conveniente identificar los matices que los diferencian entre sí.
Para tal fin, en un primer momento se analizan las definiciones que proporciona la Real Academia Española (2019) sobre estos conceptos y se observa que, mientras que el término multicultural ${ }^{2}$ alude a la convivencia de ciudadanos entre diversas culturas, la interculturalidad remite a la relación de personas con distintas culturas. De forma breve, aquí se vislumbran los aspectos principales que los diferencian y que se desarrollan a continuación.

En primer lugar, la multiculturalidad hace referencia a ciudadanos que, teniendo diferencias culturales, ideológicas, lingüísticas, etc., comparten el mismo territorio. Esta situación, muy frecuente, representa diversidad (Porras 2010; Barabas, 2014). En dichas convivencias plurales, los fundamentos de la multiculturalidad se basan en la capacidad de reconocer, aceptar y respetar la diversidad, rechazar cualquier tipo de discriminación y promover un trato igualitario hacia todos sin excepción (Giménez, 2009).

Más allá de instar a un equilibrio social, la multiculturalidad no interfiere en las posibles relaciones de ciudadanos, ni en los cambios que se podrían originar en el lugar. Reconoce, no obstante, que la falta de vínculos supone el riesgo de motivar desigualdad (Vivas, 2015). Asimismo, teniendo en cuenta que la cantidad de ciudadanos originarios de un territorio siempre será, a priori, más grande que el número de recién llegados, la multiculturalidad distingue a un grupo cultural principal (nacidos en la zona geográfica en la que viven) de minorías culturales (inmigrantes que habitan en un territorio del cual no provienen). Respecto a los conjuntos, el primero ejerce funciones de padrino hacia el segundo manifestando un carácter protector que, a su vez, simboliza un nivel superior; García, Escarbajal Frutos y Escarbajal De Haro $(2007$, p.93) concretan que:

la multiculturalidad no habla de transformación social, sino que adopta posturas paternalistas de la mayoría hacia las minorías.

En suma, la multiculturalidad atiende y valora la existencia de la diversidad, así como la convivencia conjunta de grupos heterogéneos, pero sin remitir a interrelaciones. Su enfoque

2 La palabra multicuturalidad se define como una cualidad del témino multicutural. 
se orienta a la relación entre espacio y tiempo de expresiones culturales simultáneas (García, 2005).

La interculturalidad, en cambio, no acepta la supremacía de una cultura sino que promueve la comunicación entre grupos culturales, así como el trascender de políticas educativas encargadas de constituir unos mismos derechos para todos, comprendiendo a la sociedad como un conjunto. En este sentido, la UNES $\mathrm{CO}(2017$, p.15) determina que:

La «interculturalidad» se refiere a la presencia e interacción equitativa de diversas culturas y la posibilidad de generar expresiones culturales compartidas, adquiridas por medio del diálogo y de una actitud de respeto mutuo.

A partir de aquí, se comprende que en la interculturalidad se valora al ciudadano recién llegado, se acepta y se integra en la comunidad sin establecer jerarquías y procura que, en ningún momento, pierda su propia identidad. A su vez, sin atender a diferencias, defiende la legitimidad, fomenta la igualdad y promueve el diálogo entre los habitantes (Vivas, 2015).

Teniendo en cuenta estos aspectos, se concibe que la principal característica que distingue al multiculturalismo del interculturalismo sea la relevancia que, este último, le otorga a las relaciones sociales surgidas entre ciudadanos de origen, cultura, idioma y/o ideología diferente. Interacciones que son el pilar sobre el que sustentar una convivencia saludable en una sociedad con una ciudadanía de representada diversidad (García, 2005, p.91). Cabe señalar que el interculturalismo no escapa del multiculturalismo sino que, además de compartir sus ideas, promueve los vínculos. Giménez (2009, p. 113$)$ concreta:

\footnotetext{
Si el multiculturalismo ha puesto énfasis en la creación de sociedades de iguales y diferentes, el interculturalismo también lo hace pero añade algo más: el énfasis en la interacción positiva entre los diferentes. Los principios centrales del multiculturalismo -igualdad de trato y no discriminación, por una parte, y valoración positiva de la
}

diversidad y el respeto a la diferencia, por otralos asume también el interculturalismo, pero añade una tercera guía: el principio de unidad en la diversidad a partir de enriquecer los proyectos comunes a partir de las especificidades.

De esta forma, el término interculturalidad comprende un pensamiento que huye de la segregación y el aislamiento de grupos culturales. Para ello, adquiere especial relevancia la comunicación entre ciudadanos de rasgos diversos (Porras, 2010); diálogos e interacciones que, a su vez, evitarían la creación de guetos. En síntesis, la edificación de vínculos entre todos los ciudadanos es la base que sostiene a la interculturalidad.

Expuesto el marco de referencia, se presentan 2 de los objetivos planteados para este estudio $^{3}$, los cuales se enmarcan en un contexto educativo de gran diversidad entre el alumnado:

1. Impulsar las relaciones sociales entre personas de distintas culturas.

2. Fomentar un comportamiento apropiado en prácticas grupales.

\section{METODOLOGÍA}

Está probado que el ejercicio físico mejora la salud y la calidad de vida, no solo desde un punto de vista corporal, sino también mental y social (Barbosa y Urrea, 2018). Dicha vertiente social, denota que una actividad motriz sea un potencial recurso para fomentar la interculturalidad y, en ese sentido, la Capoeira ${ }^{4}$ será el medio para intentar impulsarla.

Mencionado este aspecto, nos situamos en una investigación de carácter cualitativo, en la cual el estudio de casos orientará la fase práctica. Las características de este método posibilitan el análisis específico de una realidad en particular (L.A.C.E., 2013; Simons, 2011 ; Stake, 1998), lo que resulta relevante en este estudio ya que se desarrollará en un contexto educativo con una representación multicultural significativa entre el alumnado. Asimismo, permite que el investigador esté presente en el proceso empírico (de forma más o menos ac-

${ }^{3}$ Este artículo solo remite a una parte del estudio, delimitado por los 2 objetivos presentados.

4 Práctica que combina artes marciales y danza con un importante bagaje histórico. 
tiva) y tenga ocasión de observar el comportamiento y la actitud de los participantes en cada situación. Flyvbjerg (2004, p.50) indica en este sentido que:

La ventaja del estudio de casos es que puede "acercarse» a situaciones de la vida real y comprobar ideas en relación directa con el fenómeno a medida que se desarrolla en la práctica.

A continuación se indica el ámbito educativo en el que se desarrollará el proceso práctico, los 2 tipos de labores que se les solicitarán a los alumnos (considerando que el diseño global estará vinculado con la Capoeira y con elementos de la interculturalidad), los informantes, las técnicas e instrumentos para recoger la información y su análisis.

\section{Ámbito Educativo}

Todos los momentos, y espacios, de carácter educativo son una oportunidad para promover que el ciudadano comprenda, acepte y respete la diversidad que existe en toda sociedad plural. Sin embargo, determinados contextos resultan más idóneos para aplicar procesos de enseñanza - aprendizaje $(E-A)$ orientados a un alumnado en concreto y en un ámbito específico. En este sentido, desde la educación informal es difícil lograr unos objetivos de tipo formativo ya que, de un modo espontáneo, irrumpe más allá del marco institucional. En cambio, debido a su carácter obligatorio y reglado, la educación formal permite diseñar estructuras didácticas orientadas a objetivos concretos, lo cual posibilita una planificación y, si es necesario, una adaptación. Por su parte, la educación no formal también posee esas ventajas, pero la asistencia es voluntaria (Larson, 2000) y se concibe para el ámbito extraescolar.

Conociendo todos los contextos, este estudio de casos se sitúa en un ámbito extraescolar multicultural debido, esencialmente, a la posibilidad de programar y ajustar las sesiones según evolución del alumnado. Si bien desde la educación formal también sería posible, ésta requiere la coordinación de varios docentes 0 , incluso, del claustro al completo. Por otra parte, en las actividades extraescolares no suele existir una calificación cuantitativa, o cualitativa, que determine el conocimiento adquirido; labor frecuente en la educación formal. Santos y Sicilia (1998, p. 14) completan sobre las actividades extraescolares que:

la convivencia social es más intensa, la motivación orienta la actuación de los agentes y la calificación no condiciona tanto el aprendizaje.

Justificada la elección, se debe de considerar que el carácter voluntario pueda alterar el número de alumnos participantes, lo cual crearía sesgos y condicionaría los resultados del estudio. Para evitarlo, tan solo se valorarán a los asistentes habituales. La asistencia voluntaria, a su vez, no justifica el desorden ni exime respetar las normas e indicaciones del responsable. No hay que obviar que las actividades extraescolares se engloban en un sistema de participación que implica reglas, propósitos y restricciones (Larson, 2000) en el que se intervendrá para gestionar cada situación que se considere oportuna.

Respecto al centro educativo, se trata de una escuela situada en un barrio al oeste del Badalona en la cual, en el año 2017 se suprimió toda actividad extraescolar debido a frecuentes conflictos entre alumnos de procedencias distintas; situación que era habitual más allá de la escuela. Por ese motivo, previo a la entrada en el campo hubo 2 reuniones con el equipo directivo a fin de presentar el estudio y la intervención práctica. Según datos del Instituto de Estadística de Cataluña, en el año 2018 el municipio de Badalona contaba con 217.741 habitantes, de los cuales 27.758 eran de nacionalidad extranjera, una cifra cercana al 12,7\%. Sin embargo, en la zona en la que está ubicada la escuela, la población inmigrante representa el $49 \%$ de los habitantes del barrio.

\section{Propuesta Didáctica}

El diseño y la programación de las sesiones extraescolares consideraron la situación del alumnado en este particular contexto multicultural, el cual era el pertinente para intentar lograr los objetivos planteados mediante la introducción de la Capoeira. Con relación a la propuesta didáctica, en primer lugar se indican 2 tipos de labores que Moneo (2014, p.252) distingue y que son la referencia para el desarrollo de contenidos: 
Actividades $\mathrm{E}-\mathrm{A} \rightarrow$ prácticas que, en la mayoría de las ocasiones, se basan en la ejecución repetida de una o varias acciones que previamente se han explicado y/o mostrado. En función del diseño, el alumnado las realizará individualmente, por parejas o mediante agrupaciones. En cualquier situación, el docente tiene la mayor parte del control.

Tarea $\rightarrow$ situaciones complejas en las que el aprendizaje cooperativo posee gran presencia. Solicitan un elevado nivel de responsabilidad, autonomía y capacidad de decisión por parte de los alumnos para poder resolverlas con éxito. Por este motivo, el control durante los procesos de resolución recae sobre el alumnado.

Se diseñan, entonces, labores de distinta dificultad que promuevan relaciones personales entre una diversidad de alumnos. En este sentido, mientras que las actividades de $E-A$ poseen un grado de complejidad menor, las tareas requieren de la activación, combinación y aplicación de diversos recursos personales (conocimientos, destrezas, etc.) para poder resolverse con éxito. Se comprende que, en procesos colaborativos, el uso de recursos generará un aprendizaje más significativo que aquéllos que provengan de un procedimiento únicamente teórico o práctico; más aún si estos se implementaran de manera efectiva en contextos multiculturales. Este nexo de ideas sobre constructos susceptibles de fomentar aprendizajes duraderos, en un contexto pluricultural, es una necesidad que García (2005, p.100) relata de la siguiente manera: jorar los procesos de aprendizaje («autoaprendizaje») permanente que habrán de utilizar a lo largo de toda la vida. Por ello, se resalta más la importancia de la adquisición de técnicas y estrategias que de conocimientos.

En este caso, no obstante, se considera oportuna una planificación de actividades para el aprendizaje básico de la Capoeira puesto que, excepto en Brasil, es una práctica poco conocida. Así, el propósito en las primeras sesiones consistirá en una enseñanza inicial (instrumentos, canciones, vestuario, origen, etc.) y en, el aprendizaje de los movimientos esenciales. Éstos, en función de la actividad, se practicarán individualmente, por parejas o en pequeños grupos. Al respecto, en las actividades colaborativas los alumnos tendrán autonomía para escoger a su/s compañero/s (respetando la cantidad que se mencione) y nadie podrá intervenir. Cada sesión concluirá con la Roda instando, ahora sí, a cambiar de compañeros para el jogo ${ }^{6}$; un espacio de entre 10 y 15 minutos (los últimos) en el que se introducirán canciones de Capoeira y se enseñará a entonar coros y a batir de palmas.

Transcurridas las primeras sesiones, en las siguientes se mantendrán actividades de $\mathrm{E}-\mathrm{A}$ con el intercalo de 3 tareas, cuyas resoluciones requerirán dedicar un tiempo que, probablemente, se extenderá más allá del horario extraescolar ${ }^{7}$. Los diseños de las tareas tienen vinculación directa con los objetivos del estudio y se presentan en el Cuadro 1.
La escuela inclusiva y orientada a la diversidad
debe tratar de que los alumnos aprendan estra-
tegias que les permitan resolver los problemas de
la vida diaria de forma cooperativa y solidaria,
creando auténticos grupos de aprendizaje, y me-

5 Distribución para el jogo: semiárculo formado por alumnos (sentados) y una línea contigua de maestros (de pie), los cuales cantan y tocan música marcando la entrada, salida y ritmo del jogo.

- La práctica de la Capoeira. Los participantes se alteman para entrar en la Roda y combinar movimientos próximos entre ellos o, en determinados casos, demibarse. El ritmo vańa según música y cánticos.

7 Este planteamiento es el idóneo para el desarrollo de tareas. No obstante, solicitar dedicación fuera del horanio extraescolar es susceptible de causar problemas con la comunidad educativa. En tal caso, se puede valorar dejar un tiempo en las sesiones para resolver las tareas o ampliar los plozos de entrega. 


\section{Cuadro 1}

Descripción de las tareas

\section{Tarea Organización}

Descripción

Tiempo

Cada uno pensará una serie de movimientos que, en una secuencia, realizará ante su compañero mientras le graba (con móvil, tablet, cámara, etc.). Luego cambiarán roles. Al término, visualizarán ambas grabaciones y decidirán cuál ha sido mejor a partir de los siguientes criterios:

\section{Secuencia grabada}

- Ritmo (acorde con la música escogida).

- Velocidad de movimientos.

- Equilibrio.

- Combinación de movimientos.

- Secuencia ordenada (aprovechar posturas).

- Grado de dificultad.

\section{Creación \\ de una Grupos de \\ revista $\quad 8-9$ personas deportiva}

\section{La función teatral}

El día previsto, cada pareja mostrará sus grabaciones y revelerá su decisión.

Cada grupo diseñará una revista deportiva basada en

la Capoeira. El grupo asignará los siguientes roles en equipos de trabajo:
- Director - Coordinador

- Editor - Diseñador

- Escritor/es artículo/s

- Periodista/s

- Fotógrafo/s

- Guionista/s
Los alumnos diseñarán y representarán una función

(obra, performance, etc.) basada en la Capoeira que, como mínimo, conste de:

- Bienvenida y presentación.

- Muestra de movimientos aprendidos.

5 semanas

3 semanas

Extracurricular 
El conjunto de actividades y tareas que combina la propuesta didáctica tiene una duración de 6 meses y comprende 42 sesiones a desarrollar entre el mes de enero y junio del año 2018. Cada sesión será de 1 hora y 30 minutos, martes y viernes de cada semana (excepto días festivos). Así, la primera sesión práctica es el día 6 de enero y la última (la función teatral para alumnado, profesorado y familias de alumnos participantes) el 22 de junio. Respecto a la orientación metodológica, se concibe que la propuesta didáctica sea más apropiada para los alumnos situados entre 10 y 12 años debido, en esencia, a que la ejecución de algunos movimientos requiere una mínima condición física y, por su parte, la resolución de las tareas demanda un cierto nivel cognitivo que, se considera, está más cercano a los sujetos que se encuentran en $6^{\circ}$ (y último) curso de Primaria.

\section{Informantes}

En primer lugar, Kazez (2009) expresa como prioridad la acción de escoger a los sujetos adecuados para obtener respuestas afines a la investigación en concreto, en lugar de pretender la generalización de resultados. Por esta razón, la diversidad del alumnado, la concreción de la edad y la realidad social que enmarcaba a la escuela, fueron factores determinantes para su elección. De ese modo, el modelo restringido de informantes hizo que la muestra fuese pequeña y no aleatoria (Salamanca y Martín-Crespo, 2007, p. 1), u orientada hacia la información (Flyvbjerg, 2004, p.45).

Por su parte, la propuesta didáctica fue desarrollada entre 2 (por aquel entonces) alumnos universitarios que cursaban la mención de Educación Física en el $4^{\circ}$ (y último) curso del Grado de Magisterio de Educación Primaria. Un estudiante contaba con 2 años de experiencia como monitor de Capoeira, mientras que su compañero hacía 4 años que era alumno en una academia especializada. Ambos se encargaron de dirigir las sesiones, posibilitando al investigador realizar una observación no participante. Se consideró que, mantener una cierta distancia, facilitaría un análisis objetivo de las evidencias obtenidas.

Como se indicó, la voluntariedad de acudir a las sesiones extraescolares hizo que únicamente se consideraran informantes a los alumnos que asistieron de forma habitual. Respecto a la posibilidad de modificar, o adaptar, el número de los informantes durante un proceso de la investigación cualitativa, Martínez-Salgado (2012, p.617) indica:

\footnotetext{
Otra característica de este tipo de procedimiento es que el tamaño de la muestra no se conoce al inicio, sino solo cuando la indagación ha culminado.
}

En este sentido, al término de la propuesta didáctica se constató que 27 alumnos asistieron de manera continua a las sesiones ${ }^{8}$, en concreto 12 del grupo A y 15 del B. Con relación a la diversidad del grupo-clase, el Cuadro 2 muestra los datos relativos al país de origen, el espacio de tiempo que reside en la ciudad' y el conocimiento de algún idioma utilizado por los habitantes 1011

8 Salvo excepciones puntuales y justificadas.

- Se consideran "Recién llegados" los que se incorporaron a la escuela una vez empezado el curso y con "Menos de 1 año" aquellos que, sin residir ese tiempo en el país, iniciaron el curso.

10 En el municipio son de uso común el catalán y el castellano.

"Se agradece aquí la predisposición y ayuda del equipo directivo para la consulta de expedientes. 
Cuadro 2

Diversidad del grupo-clase

\begin{tabular}{|c|c|c|c|}
\hline Alumno & País de origen & Periodo de residencia & Conocimiento y uso del idioma \\
\hline A1 & Pakistán & Entre 1 y 2 años & $\begin{array}{l}\text { Comprende el idioma, pero no lo } \\
\text { habla o tiene muchas limitaciones }\end{array}$ \\
\hline B2 & India & Recién llegada & $\begin{array}{l}\text { No comprende el idioma o solo } \\
\text { reconoce palabras concretas }\end{array}$ \\
\hline C3 & China & Recién llegada & $\begin{array}{l}\text { No comprende el idioma o solo } \\
\text { reconoce palabras concretas }\end{array}$ \\
\hline D4 & Pakistán & Más de 2 años & $\begin{array}{c}\text { Comprende el idioma y logra } \\
\text { conversar }\end{array}$ \\
\hline E5 & Marruecos & Más de 2 años & $\begin{array}{c}\text { Comprende el idioma y logra } \\
\text { conversar }\end{array}$ \\
\hline F6 & Marruecos & Entre 1 y 2 años & $\begin{array}{l}\text { Comprende el idioma, pero no lo } \\
\text { habla o tiene muchas limitaciones }\end{array}$ \\
\hline G7 & India & Menos de 1 año & $\begin{array}{l}\text { No comprende el idioma o solo } \\
\text { reconoce palabras concretas }\end{array}$ \\
\hline H8 & Barcelona & Nativo & $\begin{array}{c}\text { Comprende y habla el idioma } \\
\text { perfectamente }\end{array}$ \\
\hline 19 & Tailandia & Menos de 1 año & $\begin{array}{l}\text { No comprende el idioma o solo } \\
\text { reconoce palabras concretas }\end{array}$ \\
\hline $\mathrm{J} 10$ & Filipinas & Entre 1 y 2 años & $\begin{array}{l}\text { Comprende el idioma, pero no lo } \\
\text { habla o tiene muchas limitaciones }\end{array}$ \\
\hline K11 & Ecuador & Más de 2 años & $\begin{array}{c}\text { Comprende y habla el idioma } \\
\text { perfectamente }\end{array}$ \\
\hline L12 & Marruecos & Más de 2 años & $\begin{array}{l}\text { Comprende el idioma y logra } \\
\text { conversar }\end{array}$ \\
\hline M13 & Barcelona & Nativo & $\begin{array}{l}\text { Comprende y habla el idioma } \\
\text { perfectamente }\end{array}$ \\
\hline N14 & Pakistán & Más de 2 años & $\begin{array}{c}\text { Comprende el idioma y logra } \\
\text { conversar }\end{array}$ \\
\hline 015 & China & Menos de 1 año & $\begin{array}{l}\text { Comprende el idioma, pero no lo } \\
\text { habla o tiene muchas limitaciones }\end{array}$ \\
\hline P16 & India & Menos de 1 año & $\begin{array}{l}\text { No comprende el idioma o solo } \\
\text { reconoce palabras concretas }\end{array}$ \\
\hline Q17 & Barcelona & Nativa & $\begin{array}{l}\text { Comprende y habla el idioma } \\
\text { perfectamente }\end{array}$ \\
\hline $\mathbf{R} 18$ & China & Recién llegada & $\begin{array}{l}\text { No comprende el idioma o solo } \\
\text { reconoce palabras concretas }\end{array}$ \\
\hline S19 & Ecuador & Más de 2 años & $\begin{array}{c}\text { Comprende y habla el idioma } \\
\text { perfectamente }\end{array}$ \\
\hline T20 & India & Menos de 1 año & $\begin{array}{l}\text { No comprende el idioma o solo } \\
\text { reconoce palabras concretas }\end{array}$ \\
\hline
\end{tabular}




\begin{tabular}{|c|c|c|c|}
\hline Alumno & País de origen & Periodo de residencia & Conocimiento y uso del idioma \\
\hline U21 & Pakistán & Más de 2 años & $\begin{array}{l}\text { Comprende el idioma y logra } \\
\text { conversar }\end{array}$ \\
\hline V22 & Rusia & Recién Ilegada & $\begin{array}{l}\text { No comprende el idioma o solo } \\
\text { reconoce palabras concretas }\end{array}$ \\
\hline W23 & Marruecos & Entre 1 y 2 años & $\begin{array}{l}\text { Comprende el idioma, pero no lo } \\
\text { habla o tiene muchas limitaciones }\end{array}$ \\
\hline $\mathrm{X} 24$ & Filipinas & Más de 2 años & $\begin{array}{l}\text { Comprende el idioma y logra } \\
\text { conversar }\end{array}$ \\
\hline Y25 & Barcelona & Nativo & $\begin{array}{c}\text { Comprende y habla el idioma } \\
\text { perfectamente }\end{array}$ \\
\hline Z26 & Pakistán & Entre 1 y 2 años & $\begin{array}{l}\text { Comprende el idioma, pero no lo } \\
\text { habla o tiene muchas limitaciones }\end{array}$ \\
\hline$\alpha 27$ & Marruecos & Más de 2 años & $\begin{array}{l}\text { Comprende el idioma y logra } \\
\text { conversar }\end{array}$ \\
\hline
\end{tabular}

Fuente: Elaboración propia

Además de alumnos y monitores, el conjunto de informantes implicó a la tutora de cada grupo-clase de $6^{\circ}$ curso y al maestro de Educación Física de la escuela (figuras que conocían a todos los alumnos).

\section{Técnicas E Instrumentos}

La observación no participante limitó la interacción con el alumnado y facilitó la objetividad en el análisis posterior. Sin perjuicio de ello, la presencia del investigador fue aceptada previamente por el grupo-clase, como se requiere en este tipo de observación (Angrosino, 2012 , p.38). Con las anotaciones se recogían evidencias como la aceptación o el rechazo a la Capoeira, la formación natural de grupos según un determinado patrón, las reacciones y comportamientos en estas asociaciones, etc.

Para completar y ajustar estas evidencias, cada sesión se registró desde 2 ángulos opuestos colocando una cámara a lados opuestos del espacio de práctica, lo que permitió observar la actuación del alumnado. Con el propósito de estructurar toda la información procedente de la observación, se elaboró en un diario de campo.

Finalmente, aunque durante el desarrollo de la propuesta didáctica fue recurrente dialogar e intercambiar información con los docentes sobre diferentes aspectos, una vez terminadas las sesiones se entrevistó a la tutora de cada grupo, al maestro de Educación Física de la escuela y a los 2 monitores; en este último caso realizándose conjuntamente debido a disponibilidades. Cada entrevista disponía de un guión semi-estructurado que incidía sobre los aspectos fundamentales del estudio y permitía ampliar una información cuando se consideraba oportuno. Si bien, el interés hacia elementos relacionados con los objetivos de la investigación establecía que las entrevistas eran focalizadas (Flick, 2004, p.89). En cuanto al diseño y formulación de preguntas, se tuvieron como referencia las aportaciones de Angrosino (2012), Stott y Ramil (2014), y Troncoso y Amaya (2017).

Con respecto a las preguntas vinculadas con los objetivos, estas se diversificaban en 2 grandes bloques que se complementaron con las anotaciones del diario de campo:

- Asociación entre los compañeros; reflexiones acerca de la reacción de los alumnos cuando se plantean labores conjuntas, así como sus respuestas al conocer quién/es será/n su/s compañero/s de trabajo.

- Comportamientos en la realización de labores en conjunto; valoraciones rela- 
tivas a la interacción entre compañeros, implicación personal durante el desarrollo de la labor e interés por conseguir una resolución exitosa.

Acotada la información procedente de cada instrumento, se realizó su análisis.

\section{Análisis De Información}

Las anotaciones en el diario de campo concretaban las evidencias observadas en cada sesión. Cada entrevista se transcribió y se hizo llegar al entrevistado para que fuese validada; teniendo así oportunidad de ratificar, o de corregir, la respectiva transcripción. Corroboradas estas, la información se incorporó al programa informático Nudist Vivo (N-Vivo) para realizar un análisis inductivo. Estructurados e interpretados los datos, se organizaron 2 categorías vinculadas a los objetivos del estudio y, en la segunda de estas, 2 subcategorías más, como se muestra a continuación:

- Relación entre sujetos de diferentes rasgos culturales; información sobre predisposición (rechazo, aceptación, indiferencia, etc.) para asociarse con personas de distinto país, cultura, idioma, etc.
- Desarrollo de labores en conjunto; evidencias sobre su actitud:

- Hacia los compañeros: respeta a la persona sin distinción, acepta opiniones distintas a las propias, muestra empatía, dialoga, llega a acuerdos, etc.

- Personal: participa, colabora, es responsable, etc.

En síntesis, la información incluida en cada apartado procedía de las evidencias del diario de campo y de las respuestas de cada entrevistado.

\section{RESULTADOS}

La muestra de resultados se ha estructurado siguiendo el orden de los 2 objetivos expuestos, los cuales se corresponden con la información recogida en cada categoría. Se debe mencionar que solo se realizaron 39 de las 42 sesiones diseñadas debido, en primer lugar, a una excursión cuya llegada fueron las 19:00 horas $y$, en segundo lugar, a que las colonias de fin de curso ausentaron 4 días (de lunes a jueves) a los alumnos de $6^{\circ}$ curso. La distribución de sesiones, entonces, quedó configurada como muestra el Cuadro 3.

\section{Cuadro 3}

Distribución de sesiones

\begin{tabular}{cc}
$\begin{array}{c}\text { Actividades de enseñanza - aprendi- } \\
\text { zaje }\end{array}$ & Explicación y desarrollo de Tareas \\
\hline Sesiones 1 a 9 & Tarea $1-$ Sesión $10^{a}$ \\
\hline Sesiones 11 a 16 & Tarea $2-$ Sesión $17^{a}$ \\
\hline Sesiones 18 a 26 & Tarea $3-$ Sesión $27^{a}$ \\
\hline $\begin{array}{c}\text { Sesiones } 28 \text { a } 38 \text { (las sesiones } 35-36- \\
37 \text { y } 38 \text { se dedicaron exclusivamente a los } \\
\text { ensayos de la función teatral) }\end{array}$ & Función teatral - Sesión 39a \\
\hline
\end{tabular}

El análisis de la información reveló los siguientes resultados:

\section{Objetivo 1. Impulsar las relaciones sociales entre personas de distintas culturas.}

Haciendo efectiva la directriz de proporcionar autonomía, en el inicio se observaron las asociaciones que formaban los alumnos (de manera natural) cuando se les solicitaba una unión, así como su actitud según quién era su/s compañero/s. Se advirtió que la mayo- 
ría de ellos siempre tenía tendencia a juntarse con los mismos compañeros y, cuando no era posible, se exteriorizaba un malestar, enfado e, incluso, algunos abandonaron la práctica renegando de alumnos y monitores; comportamientos reiterados en los sujetos D4, E5, L12, U21, W23, Z26 y a27. En este sentido, se apreció que el país de origen y el idioma común eran los primeros factores por los que se agrupaban, quedando en segundo plano el periodo de residencia, así como el conocimiento y uso del idioma. La tutora del grupo A (TG-A), del grupo B (TG-B) y el maestro de Educación Física (MEF) valoraron que:

TG-A: "Ellos tienen muy claro con quiénes se van a juntar incluso antes de empezar el curso... al menos antes era así, ahora es verdad que me tienen un poco... desconcertada (risas), pero ellos siempre se han juntado mucho en función de su idioma, de su país... piensa que hablar el mismo idioma hace mucho, uno de los que al principio lo pasó peor aquí fue M13, lo pusimos aquí por circunstancias del momento, pero fue difícil para él".

TG-B: "No se relacionaban con nadie que no fuera de su entorno, desde el principio del curso los chinos con los chinos, los marroquíes con los marroquíes y así... y al cabo de un mes ya no querían saber nada del resto, los de aquí también ¿̇eh? aquí Y25, Q17, H8, (menciona a 2 alumnos más de Barcelona no asistentes a la actividad extraescolar) siempre iban por su lado".

MEF: "Se juntaban los que hablaban igual, pero es normal... vienes aquí, no conoces a nadie y al que primero te acercas es al que te entiende, lo que pasa es que estos tampoco hacían nada luego por estar con otros, más bien al contrario, y mira que yo siempre les hacía cambiar de compañeros en los ejercicios, pero montaban unos líos entre ellos que al final pensé que no valía la pena".

Durante las primeras semanas, los alumnos repetían las agrupaciones tanto en las actividades como en la Roda; participando siempre en esta con los mismos compañeros si estaban disponibles y querían. Sin embargo, en la Roda de la $6^{a}$ sesión se observó que los alumnos Al y N 14 (pareja muy recurrente), participaron con distintos compañeros. La situación ocurrió cuando $\mathrm{H} 8$ hizo gestos a N14 para jogar con él, y este asintiera. De forma inmediata, A1 hizo lo mismo con Y25 y, segundos después, estaban juntos en la entrada de la Roda. En el registro en vídeo se advirtió que se generaron murmullos entre el grupo-clase y que diversos alumnos mostraron cierto asombro.

Durante la $7^{a}$ sesión no surgieron nuevas formaciones ni en actividades de $E$-A ni en la Roda. Sin embargo, en la Roda de la $8^{a}$ sesión, de nuevo se asociaron alumnos que nunca antes se habían unido (solo se juntaron con una persona distinta en la ocasión en que no les fue posible unirse a su compañero natural, pero lo hicieron evidenciando enfado y provocando a su pareja). Sobre estas asociaciones, la primera ocurrió entre los alumnos X24 y J10, cuando el primero le hizo gestos al segundo para salir a jogar y este negó con la cabeza. Transcurridos unos instantes en que X24 intentó, sin éxito, pedirle explicaciones, finalmente optó por preguntarle al alumno S19 el cual, sentado en el lado izquierdo de J10, había seguido lo sucedido. El alumno S19 evidenció su predisposición asintiendo inmediatamente y, en escasos minutos, ambos formaron pareja para jogar.

La segunda asociación ocurrió de un modo más natural entre las alumnas Q17 y B2. La alumna B2, llegada de la India hacía pocos meses, solo estaba con 3 compañeros procedentes del mismo país y, cuando no era así, mostraba su malestar (sin provocar al compañero). La organización en la Roda, no obstante, la situó al lado de Q17 que, por gestos, le insinuó formar pareja para jogar. La grabación en vídeo reveló que B2 mostró confusión sin, al parecer, entender exactamente qué le expresaba su compañera. Cuando una de las habituales parejas salió a jogar, la alumna Q17 llamó de nuevo la atención de B2 señalándole hacia la pareja y manifestándole la idea anterior. B2, con cierta sorpresa, aceptó. A partir de esa sesión se observó que B2 no rehusó unirse con otros compañeros para realizar cualquier labor; un ligero cambio de actitud que generó cierta discrepancia entre monitores (M-1 y M-2), y del cual advirtió su tutora.

M-1: "Lo que le pasaba a B2 es que no podía hablar con nadie que no fuera T20, P16 ó G7, y como ellos tampoco conocían el idioma de aquí, no daban el paso para hablar con otros".

M-2: "Yo creo que va más por el tema cultural. A otros les pasa lo mismo pero son más echaos 
pa'lante, otra cosa es que no se quieran juntar con los que no son de su gueto, pero estos que vienen de la India... yo creo que allí son así... les falta voluntad, o son más precavidos".

M-1: "Pero en el caso de B2 no creo que fuera por falta de carácter, cuando tuvo que estar aquel día con K11, al principio, se puso como una fiera y pasaba del chaval".

M-2: "En cuestión de enfadarse sí, pero me refiero a que no tienen ese... arrojo para ser ellos los que den el primer paso para acercarse a alguien que no sea de su país o que no hable su idioma".

M-1: "Es que eso también puede ser por un tema de carácter".

M-2: "Claro pero tu carácter está ligado a tu cultura".

TG-A: "También me sorprendió lo de B2. Aquí en clase era imposible que estuviera con alguien que no fuera G7, T20 ó P16, y al decirme tú aquello (referencia a lo expuesto en la $7^{a}$ sesión) la puse con M13 para que hicieran un comentario de texto y no dijo nada, se levantó, se puso con él y estuvieron bien".

Más allá de apreciar que los alumnos L12 y a27 seguían optando por enfadarse y abandonar la práctica cuando, en ciertas actividades, no se podían unir a los compañeros que querían, en las sesiones inmediatamente posteriores se observó que los alumnos D4, E5, U21, W23 y Z26 continuaban mostrándose inconformes pero, en cambio, realizaban las actividades cada vez con mejor actitud o, en el peor de los casos, indiferencia. Por su parte, el resto del alumnado mantenía su predisposición para agruparse con compañeros distintos, cesando en gran parte sus quejas.

Respecto al origen de nuevas asociaciones (formadas por alumnos distintos a los citados) existe un salto de tiempo hasta la $13^{a}$ sesión. En esta, se planteaba una actividad para practicar la vertical en grupos de 3 personas, de tal manera que una realizaba el movimiento en una colchoneta con la ayuda adecuada de sus 2 compañeros, los cuales permanecían a ambos lados por seguridad. Tras 3 intentos, cambiaban las posiciones. La asistencia a esta sesión y la distribución de los alumnos en esta actividad hicieron que el sujeto $F 6$ no se pudiera juntar con E5, L12, W23 ó a27 (sus inseparables compañeros) y debiera agruparse con los alumnos K11 y V22. Respecto a estos, nunca evidenciaron rechazo para unirse a cualquier persona en actividades o tareas. Sin embargo, aunque de forma moderada, sí era un comportamiento frecuente en F6. Así, cuando advirtió de esta situación mostró su disconformidad pero, durante el desarrollo de la actividad, modificó su actitud e incluso, a pesar de sus limitaciones con el idioma en común, intentó ayudar al alumno K11 a explicarle a la alumna V22 en qué consistía la actividad. Más tarde, en la Roda, formó pareja con M13 para jogar. A partir de esta sesión, se apreció que F6 no mostró enfado ni rechazo hacia los demás compañeros.

Con relación a este cambio de actitud en F6, el maestro evidenció cierta sorpresa y, curiosamente, este no fue un hecho extraño para su tutora ni en ambos monitores.

MEF: "(...) me pareció raro lo que me dijiste ese día sobre F6 (en referencia al posible cambio de actitud del alumno) y a la semana siguiente lo puse con otros compañeros (no asistentes a la actividad extraescolar) y no dijo nada, se puso con ellos sin quejarse... sin caras raras ni nada".

TG-B: "F6 no ha tenido nunca un comportamiento tan desagradable como los otros que decíamos. Sí que le daba rabia no ir con los suyos, pero tampoco me extraña que ahora esté con otros sin mucho problema".

M-1: "Lo comentamos un día nosotros, que pensábamos que no sería tan difícil que F6 se juntara con otros. Se tenía que dar la situación".

M-2: "Lo que pasa es que al no hacer nosotros los grupos, no han surgido algunas cosas que hubiesen podido surgir. Por ejemplo, si yo hubiese hecho grupos, parejas y demás, hubiese juntado a muchos que no querían ni verse. Aunque se hubiesen enfadado, yo los habría juntado".

M-1: "Yo no soy tan tan tan... porque teníamos niños que cuando no iban con quienes querían, no es que se enfadaran, es que se enfadaban y se iban allí (señala un espacio de la pista).

En cuanto al resto del alumnado, C3, O15 y R18 fueron juntas en todo momento. Si bien, 
en una ocasión en que la distribución inicial del grupo-clase las hizo separar, su actitud fue neutra (no manifestaron aceptación ni rechazo). Situación similar ocurrió con G7, P16 y T20, los cuales, al igual que B2, siempre intentaban juntarse. Sin embargo, y con la excepción de B2 en las primeras semanas, no mostraban rechazo en las ocasiones en que debieron unirse con otros compañeros. Por su parte, la alumna 19 siempre estuvo dispuesta a asociarse con cualquier compañero sin problemas. Respecto a los demás, no se detectaron nuevas agrupaciones.

\section{Objetivo 2. Fomentar un comportamiento apropiado en prácticas grupales.}

Los desarrollos y resoluciones de cada tarea fueron clave para obtener información acerca de este objetivo. La aplicación de recursos individuales en un objetivo común incidía sobre comportamientos de: responsabilidad, respeto, participación, diálogo, colaboración, etc.

Se observó que la implicación personal en labores conjuntas se equiparaba, en la mayoría de casos, con la reacción manifestada al conocer la persona, o grupo, con el que haría la tarea. Así, cuando el alumno aceptaba a su/s compañero/s, su actitud era óptima en el desarrollo y, con ciertas excepciones, la resolución era exitosa. En cambio, cuando expresaba rechazo (por tener que unirse a alguien de otro país o con el que no compartía el idioma) su actitud era disidente e improductiva.

En la Tarea 1, la distribución autónoma originó una formación de 12 parejas y de 1 trío cuyas asociaciones, en su mayoría, fueron entre compañeros habituales. Por lo que hubo conformidad del grupo con la excepción del alumno E5, procedente de Marruecos, y del alumno U21, originario de Pakistán. En las primeras sesiones, ambos abandonaron la práctica evidenciado gran enfado al no poder con unirse, respectivamente, el alumno L12, W23 ó a27 (Marruecos), y D4 ó Z26 (Pakistán). En cambio, a partir de la $9^{a}$ sesión mostraron predisposición para unirse con cualquier persona; cambio de comportamiento que no surgió en el alumno E5 (cuando advirtió que formaría pareja con la alumna V22) y tampoco en U21 (al conocer su asociación con Y25).

El resultado de la tarea en ambas parejas no fue exitoso. Los alumnos E5 y U21, no obstante, reconocieron que no habían colaborado en el diseño de la secuencia. Por su parte, el alumno Y25 expresó su frustración por tener un compañero con el que no había podido hacer nada debido a su falta de interés, lo que provocó su desconexión con él. La alumna V22, gracias a la voluntad y el esfuerzo de los monitores, logró comprender qué eran las tareas y en qué consistía esta. Sin embargo no expuso reflexiones ya que, recién llegada de Rusia (y sin conocimiento del idioma), le resultaba prácticamente imposible comunicarse. Los monitores manifestaron respecto a la Tarea 1:

\footnotetext{
M-2: "Yo pienso que trabajaron bien ya en la primera tarea, lo que pasa es que en esa también hicieron parejas los mismos de siempre".
}
M-1: "No en todas (se dirige a M-2) ¿̇te acuerdas qué pasó con E5 y U21 cuando vieron que no podían juntarse con... los suyos? (entrecomilla con los dedos la última parte)".
M-2: "U21 se quedó con Y25... se cabreó y no hicieron nada... E5 se fue con V22, pero pasó literalmente de ella... y ella no dijo nada porque no sabía".
M-1: "Pero reconocieron que había sido culpa suya".

Para la creación de la revista en la segunda tarea, los alumnos debían organizarse en grupos de 8 ó 9 personas, lo cual generó que se unieran sujetos con orígenes, culturas e idiomas distintos. El Cuadro 4 muestra la distribución de los 3 grupos formados.

\section{Cuadro 3}

Distribución de sesiones

\section{Grupo1 Grupo2 Grupo3}

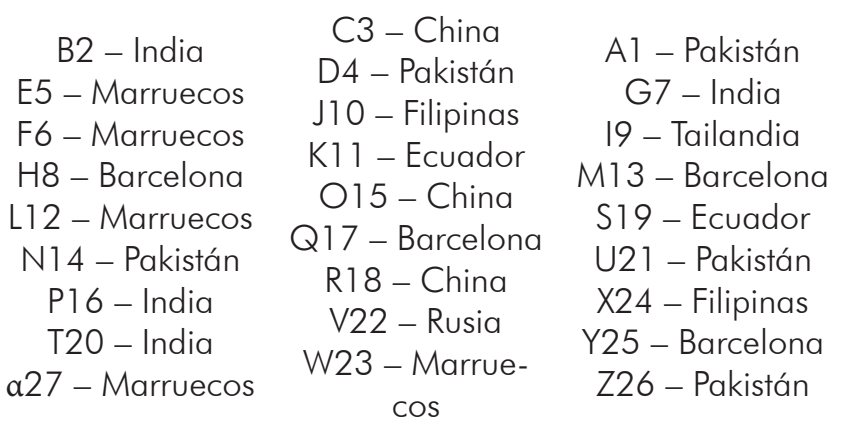


El resultado final de las revistas fue positivo y, ciertamente, se apreció que todos los grupos lograran resolver la tarea con éxito. Presentadas las 3 revistas, se preguntó al alumnado sobre el comportamiento de los compañeros durante los procesos. Con mayor y menor dificultad, todos expresaron su opinión al respecto. En este sentido, en el grupo 1 se reveló que E5, F6, L12 y $\square 27$ no habían aportado nada en las primeras reuniones. Sin embargo, transcurridos unos días, E5 y F6 se empezaron a implicar. En cambio, L12 y $\square 27$ nunca colaboraron y, cuando se juntaban, ridiculizaban al que decía algo (siempre que no fuera E5, F6 ó H8). Respecto a N14, coincidieron en que fue el más preocupado por hacer la revista. Por su parte, indicaron que B2, T20 y P16 no aportaban ideas, pero respetaban lo que decían sus compañeros e hicieron la labor individual que se les pidió.

Situación similar a esta última se produjo en el grupo 2 con las alumnas C3, O15 y R18. Varios compañeros expresaron que solo hablaban entre ellas y que no intentaban decir algo cuando se reunían. No obstante, para sorpresa del resto del grupo, el alumno K1 1 y la alumna Q17 afirmaron que una vez habían ido juntas a enseñarles las fotos que habían hecho para una sección de la revista (esta era su labor) y a preguntarles lo que no habían entendido, lo cual confirmaron las 3 alumnas. Debido al idioma, se mencionó la dificultad de V22 para poder dialogar con sus compañeros. Sin embargo, revelaron que se lograron comunicar utilizando el traductor de un móvil. En cuanto a los alumnos D4, J10 y W23, la alumna Q17 señaló que no participaron al principio pero que, después de unos días y a pesar de las dificultades de J10 y W23 con el idioma, aportaron ideas.

Con relación al grupo 3, los alumnos M13, S19, X24 e Y25 indicaron que habían tenido dificultades para comunicarse con A1, G7 e 19 a causa del idioma. Puntualizaron que, aun así, desde el principio escuchaban e intentaban saber qué debían hacer. Con el transcurso de los días, consiguieron expresar algunas opiniones y dialogar con el resto. Sobre los alumnos U21 y Z26, se afirmó que en los primeros días no habían colaborado y que en una ocasión en que X24 estaba hablando, se rieron de él sin motivo aparente. A partir de la segunda semana la actitud de ambos cambió y empezaron a dialogar, aportar ideas, etc. Los propios alumnos admitieron que había sido así. Finalmente, se reconoció la implicación de Z26 mencionando la dificultad que tenía con el idioma. Aludiendo a esta segunda tarea, tutoras y maestro de EF hicieron las siguientes reflexiones acerca de la actitud de sus alumnos:

TG-A: "He notado cambios en D4, U21 y Z26 porque, es que no hacían nada. Era una discusión constante con ellos, y la verdad es que poco a poco sí que he ido viendo que participan más... En aquello de la revista no me esperaba que hicieran nada. Yo les iba preguntando a algunos cómo lo llevaban y eso... al principio me decían que este y aquel no hacían nada, que lo tenían que hacer todo ellos, que otros ni entendían lo que decían (risas)... pero recuerdo el día que me enseñaron la revista, que les pregunté cómo la habían hecho y me empezaron a decir que este había escrito esto, el otro había dibujado la portada, no sé quién las fotos, todos habían hecho algo. (...) En eso de la revista me enteré que E5 y W23 no pusieron interés al principio, pero en su caso no se me hace tan raro que acabaran colaborando y participando, no sé por qué pero no me extraña tanto, puede que por su forma de ser, su cultura, no sé".

TG-B: "Cuando estaban haciendo la revista aquella venía Q17 y me decía que las chinas iban a enseñarle unas fotos y que le preguntaban cosas, y que ella no se enteraba (risas), y un día en el patio vi que estaban las cuatro y el K1 1, del otro grupo, y fui para allá a ver si entre todos podíamos explicarles algo. Y luego lo pensé... que nunca había visto que estuvieran intentando hablar con alguien que no fuera ellas... aunque tampoco me extraña porque la C3 y la R18 (hermanas) vinieron a la mitad de curso, más perdidas que todo... y encontraron a la 015 , que le cuesta pero es un sol de niña, y ya pues no se separaron para nada. (...) L12 y a27 no tienen solución, me dijeron que en la revista no hicieron nada y que se metían con los demás... y sin embargo otros como el J10, que ya has visto que le cuesta expresarse, o la V22, que no entiende nada de castellano y menos de catalán, sé que se pusieron a trabajar con los demás. Algo parecido pasó también con $A 1$, lo que pasa es que este chico ha tenido la suerte de poder juntarse en clase con N14, le ha ido ayudando bastante porque tira mucho y es muy bueno".

MEF: "Posiblemente fue con lo de las revistas cuando se notó más cambio en algunos, sobre todo en D4, E5 y W23 del grupo A. El otro día hablando de esto con TG-A, me dijo que ella 
también lo había notado en U21 y Z26, pero yo con ellos no he visto tanta diferencia. (...) Luego está el tema de los niños de la India... y de la niña que también llegó de allí hace poco. Si ella (B2) no se ponía con G7, P16 ó T20 se enfada$\mathrm{ba}$, y creo que a esto de la Capoeira fue porque iban ellos, pero con las semanas también es verdad que empezó a estar como más predispuesta para juntarse con otros".

Finalmente, la actitud de la mayoría de los alumnos fue positiva en las 4 sesiones dedicadas a ensayar la función teatral de la Tarea 3. De forma específica, se observó que Q17, Y25, K11, H8, N1 4 y S19 mostraron gran iniciativa desde un inicio. Tras plantear la tarea, se dirigieron hacia sus compañeros para dar ideas sobre el diseño de la función. A partir de aquí, asumieron responsabilidad encargándose de organizar y gestionar a sus compañeros; con la excepción de los alumnos L12 y a27, los cuales no se implicaron en ningún momento y no asistieron el día de la función.

Por su parte, se apreció la voluntad y esfuerzo de aquellos que residían en el país desde hacía poco tiempo y que comprendían muy poco (o nada) del idioma. Esta era la situación de las alumnas recién llegadas de China (C3 y R18), Rusia (V22) e India (B2), y de los que habían llegado hacía menos de 1 año, como le ocurría a 19 (procedente de Tailandia) y a G7, P16 y T20 (originarios de India). En cuanto al resto del alumnado, no se observaron hechos significativos. Las dinámicas fueron participativas, colaborativas y respetuosas por gran parte del grupo. La valoración de las tutoras, del maestro de EF y el diálogo de los monitores sobre aspectos vinculados a esta tarea, fueron los siguientes:

TG-A: "Esto de la función ha sido (gesticula) es que ni los padres se lo creían. Hablé con los padres del M13 y me explicaban que el niño les decía que se iba a ensayar Capoeira con unos amigos. Los padres de $\mathrm{K} 11 \mathrm{me}$ dijeron que les enseñaba en casa lo que iba a hacer... la verdad es que muy bien, y sé que del otro grupo también han habido padres que le han dicho lo mismo a TG-B".

TG-B: “(...) al ver estos últimos días que estaban todos juntos en el patio hablando, que apuntaban cosas y eso, parecía que estaban tramando algo... le pregunté a TG-A y me dijo que era por lo de la función de extraescolar, yo ya ni me acordaba que me lo dijiste (risas). Entonces al día siguiente empecé a fijarme más y vi que estaban ensayando eso, vi que $\mathrm{Y} 25$ y H8 estaban organizando a los otros, bueno y $\mathrm{S} 19$ también tenía un poco la voz cantante (...) he visto que las niñas chinas se han acercado mucho a Q17 y a N14".

MEF: "En las últimas clases me preguntaron si podían ensayar la función (...) pues por un lado fueron H8, Y25, Q17 y S19 y del otro grupo... sobre todo $\mathrm{M} 13$ y $\mathrm{K} 11$ (...) a mí lo que me sorprendió es que en el patio se juntaran casi todos para hablar de eso, incluso la niña de Tailandia y la de Rusia estaban allí intentando enterarse de algo (risas)".

M-2: "(..) los que empezaron a espabilar a los otros para montar la función fueron Y25, Q17, $\mathrm{H} 8, \mathrm{~K} 11$, N1 4 y S19, si no es por ellos se quedan todos callados y no hacen nada".

M-1: "Y por M13 también, lo que pasa es que al principio él tenía un rol más secundario y parecía que solo hacía lo que le decían, pero si te acuerdas, cuando se juntaban y hablaban, él también organizaba bastante, pero no lo hacía tan exagerado como los otros (...) cuando acabó vinieron varios padres a felicitarnos, estaban contentos, los padres de los de aquí (Barcelona) vinieron todos, y luego también hablé algo con los de Filipinas porque los padres de X24 me hicieron de traductor (risas) y con los de E5 y D4 también, que me dijeron que habían visto que se juntaban con otros compañeros".

M-2: "Yo hablé con los padres de los de aquí también y con los de K11 y S19... y N14 vino con sus padres para despedirse y los dos muy bien también, me dieron las gracias y me dijeron que había estado muy interesado, que le gustaba la Capoeira, que hacía cosas con los demás... muy bien".

\section{DISCUSIÓN Y CONCLUSIONES}

La primera reflexión se vincula con la introducción de la Capoeira, una actividad desconocida para los alumnos que les generó un curioso interés. Sus motivaciones en el transcurso de las sesiones fueron distintas, prefiriendo algunos indagar sobre su historia, analizar los movimientos como formas de expresión corporal o practicar el arte marcial.

Respecto al primer objetivo, en las sesiones iniciales se observó que los alumnos se juntaban según se hablaba un mismo idioma y, cuan- 
do no era así, renegaban de todos mostrando enfado. Se constató así que, en este contexto, no existía ninguna interacción entre alumnos de culturas distintas, siendo esta la principal aportación de la perspectiva intercultural (Giménez, 2003). La diversa procedencia había originado guetos en grupos aislados entre sí. Los docentes indicaron que estos se fundaban de modo natural al inicio de cada curso y que, en el plazo de 4-5 semanas, reflejaban un carácter excluyente hacia los que no tenían la misma cultura, lengua, religión, ideología, etc.

Los docentes intentaron que los alumnos se unieran sin atender a rasgos distintos entre ellos, para lo cual proponían actividades colaborativas. Sin embargo, cada ocasión terminaba con enfrentamientos entre alumnos de países o culturas distintas. Finalmente, optaron por cesar los intentos. Se concibe, así, una carencia de la formación docente con respecto a estrategias didácticas que favorezcan el conocimiento y el respeto hacia otras culturas y las relaciones interpersonales que se puedan derivar. En este sentido, Jutinico (2017, p.283) comprende la creación de proyectos educativos globales e interculturales, avalados desde políticas educativas, que erradiquen prácticas discriminatorias.

En cuanto al diseño y aplicación de propuestas que aúnen actividades de $E$ - $A$ y tareas, se concibe que la activación y combinación de los recursos personales necesarios para resolver estas últimas, puedan promover la unión de personas aisladas en contextos multiculturales. En este estudio la Capoeira fue el medio para generar una aproximación intercultural y, con las excepciones de los alumnos L12 y a27, así ocurrió. En una de las habituales conversaciones con los docentes, se conoció que varios alumnos quedaban en un parque cercano a la escuela para practicar Capoeira; esta información se comunicó a los monitores $y$, tras preguntarles, los alumnos explicaron que una tarde se encontraron por casualidad y que empezaron a reunirse para practicar las secuencias de la Tarea 1. A partir de esa tarde, fue algo frecuente para gran parte de los asistentes habituales. Este se consideró el aspecto clave para que se empezaran a unir (sin enfadarse) con compañeros de distintas procedencias, idiomas, culturas, etc., teniendo así la oportunidad de empezar a crear relaciones sociales en la diversidad.

El segundo objetivo, referente al comporta- miento individual en un grupo multicultural, tenía un doble propósito. Por un lado, consistía en la aceptación, respeto y valoración de la diversidad y, por otro, determinaba la implicación en la conquista de un objetivo común. Los comentarios y reflexiones del alumnado finalizadas la Tarea 1 y 2, así como el desarrollo de la Tarea 3, evidenciaron cambios de actitud en varios sujetos. Un ejemplo al respecto se observó en los alumnos E5 y U21. En las sesiones iniciales, abandonaban la práctica cuando no se podían unir a los compañeros que querían y, en la Tarea 1, reconocieron su falta de colaboración. Sin embargo, aunque en un primer instante tampoco participaron en la Tarea 2, en el transcurso se implicaron en el diseño de la revista realizando sus respectivas labores. Por otra parte, alumnos como Q17, Y25, K11, $\mathrm{H} 8, \mathrm{~N} 14$ y $\mathrm{S} 19$, que en un principio tenían un rol poco activo, mostraron iniciativa y seguridad en sí mismos al ser capaces de gestionar y organizar al grupo en la Tarea 3 . En otro orden, en las Tareas 2 y 3 se comprobó que personas con dificultad en el idioma se habían esforzado por dialogar con sus compañeros recurriendo al traductor del móvil.

Respecto a la Tarea 3, en las sesiones en que se ensayó la función (de la 35 a la 38) se constató que la mayoría del alumnado escuchaba y respetaba la opinión de todo compañero aunque no compartiera las mismas ideas, sin dejar por este motivo de seguir haciendo aportaciones. Berttollini y Langón (2009, p.34) señalan que estas interacciones descubren las virtudes de los individuos y son un medio para sociabilizarse en un grupo multicultural. Una vez se entiende esta dinámica de relaciones personales, originadas en contextos multiculturales (Barabas, 2014), es factible generar procedimientos adecuados para desarrollar la interculturalidad. En este estudio, sin embargo, se obtuvieron indicios de cambios de comportamiento en varias personas, pero no se evidenció esta posibilidad en el alumno L12 ni a27. En consecuencia, no se alcanzaron los objetivos propuestos en todo el conjunto del grupo-clase.

En síntesis, se concluye que las actividades y tareas de contenido motivador para el alumno pueden favorecer una predisposición para asociarse y colaborar con cualquier compañero. La aplicación de propuestas didácticas similares, en ámbitos multiculturales cuyas personas se encuentren segregadas por diferencias étnicas, permitirá corroborar o rebatir 
este supuesto. Como afirma Martínez (2006, p.173), generalizar los resultados de un estudio de caso consiste en desarrollar una teoría que pueda transferirse a otros casos que así lo permitan.

\section{REFERENCIAS}

Angrosino, M. (2012). Etnografia y observacion participante. Madrid: Ediciones Morata.

Barabas, A. M. (2014). Multiculturalismo, pluralismo cultural y interculturalidad en el contexto de América Latina: La presencia de los pueblos originarios. Revista Configurações, Braga, 14, 11-24.

Barbosa, S. H. y Urrea, A. M. (2018). Influencia del deporte y la actividad física en el estado de salud físico y mental: una revisión bibliográfica. Revista Katharsis, Colombia, 25, 141-159.

Bernabé, M. (2012). Pluralidad, multiculturalidad e interculturalidad, conocimientos necesarios para la labor docente. Revista Educativa Hekademos, 11, 67-76. Recuperado de https://dialnet.unirioja.es/servlet/ articulo? codigo $=4059798$. [Consulta: 12 febrero 2019].

Bernabé, M. (2016). Formación para la ciudadanía intercultural en educación infantil. ¿Qué sucede en las universidades valencianas? Profesorado: Revista de curriculum y formación del profesorado, 2 (20), 328348. Recuperado de http://www.redalyc. org/articulo.oa?id=56746946018. [Consulta: 5 febrero 2019].

Berttollini M. y Langón M. (2009). Diversidad cultural e interculturalidad. Propuestas didácticas para la problematización y la discusión. Materiales para la construcción de cursos. Buenos Aires: Ediciones Novedades Educativas.

Flick, U. (2004). Introducción a la investigación cualitativa. Madrid: Ediciones Morata, A Coruña: Fundación Paideia Galiza.
Flyvbjerg, B. (2004). Cinco malentendidos acerca de la investigación mediante los estudios de caso. Revista Española de Investigaciones Sociológicas (Reis), Madrid, 106, 33-62.

García, A.; Escarbajal Frutos, A. y Escarbajal De Haro, A. (2007). La interculturalidad. Desafío para la educación. Madrid: Editorial Dykinson.

García, J. L. (2005). Educación Intercultural. Análisis y propuestas. Revista de Educación, España, 336, 89-109.

Giménez, C. (2003). Pluralismo, multiculturalismo e interculturalidad. Propuesta de clarificación y apuntes educativos. Revista Educación y Futuro: Revista de Investigación Aplicada y Experiencias Educativas, Madrid, 8, 11-20. Recuperado de https://dialnet. unirioja.es/descarga/articulo/2044239. pdf. [Consulta: 9 diciembre 2018].

Giménez, C. (2009). El Impulso de la convivencia ciudadana e intercultural en los barrios europeos: Marco conceptual y metodológico. En WAA. Marco conceptual y buenas prácticas en ciudadanía y convivencia en barrios europeos. Proyecto INTI-CIEN. Colección Documentos de Trabajo, Serie Igualdad y Ciudadanía, Barcelona, 13. Recuperado de https://wwwl diba.cat/ uliep/pdf/46644.pdf. [Consulta: 14 marzo 2019].

Instituto de Estadística de Cataluña. Generalitat de Catalunya. Recuperado de https:// www.idescat.cat/?lang=es. [Consulta: 25 abril 2019].

Jutinico, M. S. (2017). Educación para la vida: elementos de diferencia cultural para propuestas pedagógicas. Revista Infancias Imágenes, Colombia, 2 (16), 282-294.

Kazez, R. (2009). Los estudios de casos y el problema de la selección de la muestra. Aportes del Sistema de Matrices de Datos. Revista Subjetividad y Procesos Cognitivos. Argentina, 1 (13), 71-89.

L.A.C.E. (Laboratorio para el Análisis del Cambio Educativo, grupo de investigación HUM-109). (2013). Los estudios de caso. Red Universitaria de Investigación Innovación Educativa (REUNI+D), Barcelona: 
Universidad de Barcelona. Recuperado de http://hdl.handle.net/2445/33367. [Consulta: 9 febrero 2019].

Larson, R. W. (2000). Toward a psychology of positive youth development. Revista American Psychologist, Washington, 1 (55), 170 183.

Martínez, P. D. (2006). El método de estudio de caso: estrategia metodológica de la investigación científica. Revista Pensamiento y gestión. Colombia, 20, 165-193. Recuperado de http://www.redalyc.org/articulo. oa? id=64602005. [Consulta: 12 noviembre 2017].

Martínez-Salgado, C. (2012). El muestreo en investigación cualitativa. Principios básicos y algunas controversias. Revista Ciência \& Saúde Coletiva. Rio de Janeiro, 3 (17), 613-619.

Moneo, S. (2014). La Capoeira como contenido de la educación física escolar. Análisis de su incidencia educativa en un estudio de caso. Barcelona: Universidad de Barcelona. Recuperado de http://hdl.handle. net/10803/283809. [Consulta: 5 abril 2019].

Porras, J. (2010). El valor de La educación intercultural. Madrid: Editorial Visión libros.

Real Academia Española. Diccionario de la Lengua Española, 23a ed. Madrid: Editorial S.L.U. ESPASA LIBROS, 2014. Recuperado de http://www.rae.es/. [Consulta: 17 enero 2019].

Salamanca, A. B. y Martín-Crespo, Ma . C. (2007). El muestreo en la investigación cualitativa. Revista Nure investigación, 27, 1-2. Recuperado de http://www.nureinvestigacion.es/OJS/index.php/nure/issue/ view/43. [Consulta: 14 noviembre 2017].

Santos, M. y Sicilia, A. (1998). Actividades físicas extraescolares. Una propuesta alternativa. Barcelona: Editorial Inde.

Stott, L. y Ramil, X. (2014). Metodología para el desarrollo de estudios de caso. Centro de innovación en tecnología para el desarrollo humano. Madrid: UPM. Recuperado de http://www.itd.upm.es/wp-content/ uploads/2014/06/metodologia estudios de caso.pdf. [Consulta: 10 abril 2018].

Simons, H. (2011). El estudio de caso: teoría y práctica. Madrid: Ediciones Morata.

Stake, R. (1998). Investigación con estudio de casos. Madrid: Ediciones Morata.

Troncoso, C. y Amaya, A. (2017), Entrevista: guía práctica para la recolección de datos cualitativos en investigación de salud. Revista de la Facultad de Medicina, Bogotá: Universidad Nacional de Colombia, 2 (65), 329-332. Recuperado de https://doi. org/10.15446/revfacmed.v65n2.60235. [Consulta: 3 octubre 2017].

UNESCO. (2017). Textos fundamentales de la Convención de 2005 sobre la Protección y la Promoción de la Diversidad de las Expresiones Culturales. París. Recuperado de https://unesdoc.unesco.org/ark:/48223/ pf0000260710 spa. page $=16$. [Consulta: 21 enero 2019].

Vecina, C. (2013). La acción comunitaria, un camino para la integración social y la interculturalidad. Revista de Investigaciones en Intervención Social, España, 5 (3), 54-75.

Vivas, J. A. (2016). Pensando la educación desde lo plural: el 'animal educandum, ex diversitate' en el acto educativo. Revista Sophia: colección de Filosofía de la Educación, Ecuador, 18, 37-54. 